\title{
Degree of Light Damage to the Retina Varies With Time of Day of Bright Light Exposure ${ }^{1}$
}

\author{
MARY P. WHITE*2 AND LESLIE J. FISHER ${ }^{+3}$ \\ Departments of Anatomy $\dagger$ and Psychology, * The University of Michigan, Ann Arbor, MI 48109
}

Received 24 December 1986

\begin{abstract}
WHITE, M. P. AND L. J. FISHER. Degree of light damage to the retina varies with time of day of bright light exposure. PHYSIOL BEHAV 39(5) 607-613, 1987.-After adaptation to cyclic illumination (14:10 light-dark cycle; intensity $=1.4$ log $\mathrm{ftc})$ male albino rats were exposed to brighter light $(2.15 \mathrm{log} \mathrm{ftc})$ seven hours daily for 25 days. The extent of retinal damage was determined histologically. Daily exposure to bright light in the morning produced greater photoreceptor loss than daily afternoon exposure. Severe retinal damage was partially prevented by brief light adaptation ( 20 minutes duration, at $1.4 \mathrm{log}$ ftc intensity) before the onset of morning exposure. However, light adaptation did not remove the differential effect of time of day. The authors conclude that increased light exposure during the period of rod outer segment phagocytosis enhances photoreceptor damage.
\end{abstract}

Photoreceptors Retinal damage Light adaptation Light-dark cycle Albino rat Phagocytosis

\begin{abstract}
ALBINO rats exposed to bright $[13,14]$ or continuous $[21,22]$ illumination sustain irreversible photoreceptor loss. With light microscopic histological examination, the outer retinal layers appear swollen and pyknotic, and eventually become depleted [5, 22, 23]. As observed with the electron microscope, the normal "stacked-disc" appearance of rod outer segments is initially replaced in the light-damaged retina by sacs or whorls filled with elongated tubules $[9,25]$. Rod inner segments, nuclei, and synaptic terminals are also vacuolated [9]. The outer segments become increasingly disorganized, until they are phagocytosed [9]. The retinal pigment epithelium, which figures largely in theories of the mechanism of photic damage, may or may not also degenerate [14]. In contrast to the severe loss of photoreceptors, inner retinal layers remain histologically normal [13]. The degree of photoreceptor cell loss is positively related to light intensity and duration [14], and is more severe under hyperthermal conditions [14].

Hypotheses as to how light damages the retina include: accumulation of a toxic photoproduct [13] such as peroxide $[1,13]$ or vitamin $A[15]$; stereoisomeric disruption of disc membranes [9]; overtaxation of the photoreceptors' metabolic system [5]; pigment epithelium dysfunction [6,7]; interference with outer segment turnover [10]. These postulations are not mutually exclusive. For example, abnormal
\end{abstract}

retinal pigment epithelium function could impair photoreceptor nutrition, outer segment phagocytosis, and Vitamin A metabolism.

Rod outer segment disc disposal has a circadian periodicity in the rat $[11,12]$. Large phagosomes, those which are presumably recently shed outer segment discs, are dense in the retinal pigment epithelium only once each day, roughly one-to-three hours after daily light onset [11]. Bright light blocks circadian shedding of rod outer segment discs in rats [26], a response shared by other endogenous rhythms in the rat $[8,24]$. This finding forms the basis of the suggestion that disrupted outer segment membrane disposal causes photoreceptor death in light-damaged retinas [10].

The present study tested the prediction that albino rats would be more susceptible to photic damage during circadian outer segment disc disposal. Retinal damage was determined using quantitative histological techniques. We report that increased light exposure during the period of rod outer segment phagocytosis enhances photoreceptor damage.

\section{METHOD}

\section{Animals}

Twenty-eight adult, male albino rats (CD 1 strain) were

\footnotetext{
${ }^{1}$ Supported by USPHS Vision Training Grant EY07022 to M.P.W. and Research Grant EY01281 to L.J.F. from the National Eye Institute, Bethesda, MD.

${ }^{2}$ Requests for reprints should be addressed to Mary P. White, Ph.D., Department of Ophthalmology, Palo Alto Veterans Administration Medical Center, 112B1, Palo Alto, CA 94304.

3Present address: Department of Ophthalmology, Henry Ford Hospital, 2799 W. Grand Blvd., Detroit, MI 48202.
} 
TABLE

CRITERIA USED TO DEFINE SCORES OF OUTER SEGMENT

LOSS (SEE FIG. 1)

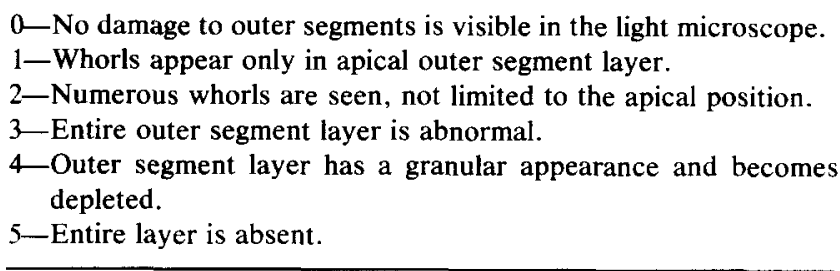

obtained from Charles River of Portage, MI. The rats had been raised at Portage on a 12:12 light-dark cycle with fluorescent light onset at 6:00 a.m. Eastern Standard Time. Upon arrival in our laboratory, the animals were adapted to the experimental 14:10 light-dark cycle, with light onset at 4 a.m. E.S.T. Light intensity in the animal room was $1.4 \mathrm{log}$ $\mathrm{ftc}$, as measured with a SEI photometer at the level of the cages. Room temperature was kept at approximately $27^{\circ} \mathrm{C}$.

Before exposure to bright light, the rats were adapted to our laboratory's light-dark cycle for 3-4 weeks. The rats were 15 weeks of age in Experiment 1 and 10.6 weeks of age in Experiment 2 at the beginning of bright light exposure.

\section{Light Exposure}

Exposure to bright illumination was accomplished by placing the animals' cages, which were open at the top and covered with a wire grating, under a rack of two 48-inch cool-white $40 \mathrm{~W}$ fluorescent bulbs. The same type of bulbs provided room lighting. "Bright" light intensity, measured with a SEI photometer, was $2.14 \log \mathrm{ftc}$ during Experiment 1, and 2.16 log ftc in Experiment 2. To allow light exposures to differ among groups in the same animal room, the bright light racks were encased in wooden boxes, $61 \mathrm{~cm}$ wide $\times 127$ $\mathrm{cm}$ long $\times 102 \mathrm{~cm}$ high, which held up to four cages. Each cage contained two or three animals. The boxes were ventilated by muffin fans, and painted white inside. Temperature was monitored and is reported in the Results section.

\section{Experimental Design}

In Experiment 1, bright light exposure was superimposed on the established photoperiod, so as not to alter the duration of daily illumination, nor the time of light onset or offset. "Morning" light exposure was from 4:00 a.m. to 11:00 a.m. "Afternoon" light exposure was from 11:00 a.m. to 6:00 p.m. ( 5 animals in each treatment group). For the remainder of the light portion of the light-dark cycle, the rats were in the normal room lighting. They were sacrificed at the end of 25 days of this treatment. Their eyes were removed and the retinas were examined histologically to ascertain the extent of photoreceptor damage and loss.

In Experiment 2, Experiment 1 was replicated (4 rats in each treatment group). In addition, 6 rats which had never been exposed to bright light were sacrificed at the same times as the exposed rats to control for possible effects of time of day of sacrifice.

Because the morning bright light exposure began simultaneously with the onset of light in the animal housing rooms, the animals exposed to bright light in the morning were dark-adapted at the beginning of the bright light exposure. The animals exposed in the afternoon, however, were light- adapted to room illumination at the onset of daily bright light exposure. To control for effects of light adaptation, 4 rats were light-adapted to room illumination for 20 minutes before a daily morning light exposure. For the light-adapted group, the exposure to bright light began at $4: 20 \mathrm{a} . \mathrm{m}$. and lasted until 11:20 a.m.

All the animals exposed to bright light were sacrificed immediately after the final light exposure. All the rats were light-adapted at sacrifice.

\section{Histology}

The animals were sacrificed by Chloropent anesthesia overdose and perfused intracardially with trialdehyde fixative (3\% glutaraldehyde- $2 \%$ formaldehyde-1\% acrolein $-2.5 \%$ dimethyl sulfoxide buffered in sodium cacodylate) with $0.1 \%$ procaine hydrochloride. Eyes were processed for light and electron microscopy according to previously published procedures [4]. Samples were taken from the horizontal meridian of the posterior retina. Horizontal (transverse) sections $1 \mu \mathrm{m}$ in thickness were obtained on an ultramicrotome (LKB Instruments, Inc.), stained with methylene blue, and examined on a Zeiss research microscope. Ultrathin horizontal sections were obtained on the same ultramicrotome, stained with uranyl acetate and lead citrate, and examined on a Hitachi 11 A electron microscope. A calibration grid, Fullam No. 1002, was photographed with each group of pictures.

\section{Data Collection}

Morphological measurements were made, using a filar micrometer, approximately $125 \mu \mathrm{m}$ from the exit of the optic nerve. Light damage to the retina was quantified by measuring on $1 \mu \mathrm{m}$ sections the outer nuclear layer and the combined outer and inner segment layers. In addition, in Experiment 2 , the following morphological features were measured: thickness of the entire retina, including the retinal pigment epithelium; the optic fiber layer; the retinal pigment epithelium, with and without its apical projections; and number of photoreceptor nuclei in the vertical extent of the outer nuclear layer. The appearance of the retinal pigment epithelium, i.e., presence of vacuoles or other abnormalities, was noted in all retinas.

The extent of damage was quantitatively assessed by scoring an ordinal variable based upon the light microscopic appearance of the retina, "Outer Segment Loss." Scores of Outer Segment Loss ranged from a minimum of 0 to a maximum of 5 , defined according to the criteria listed in Table 1.

The vertical extent of the inner plexiform layer was also measured, in part as a check on orientation, because no damage effects have been reported in this layer. Data were 


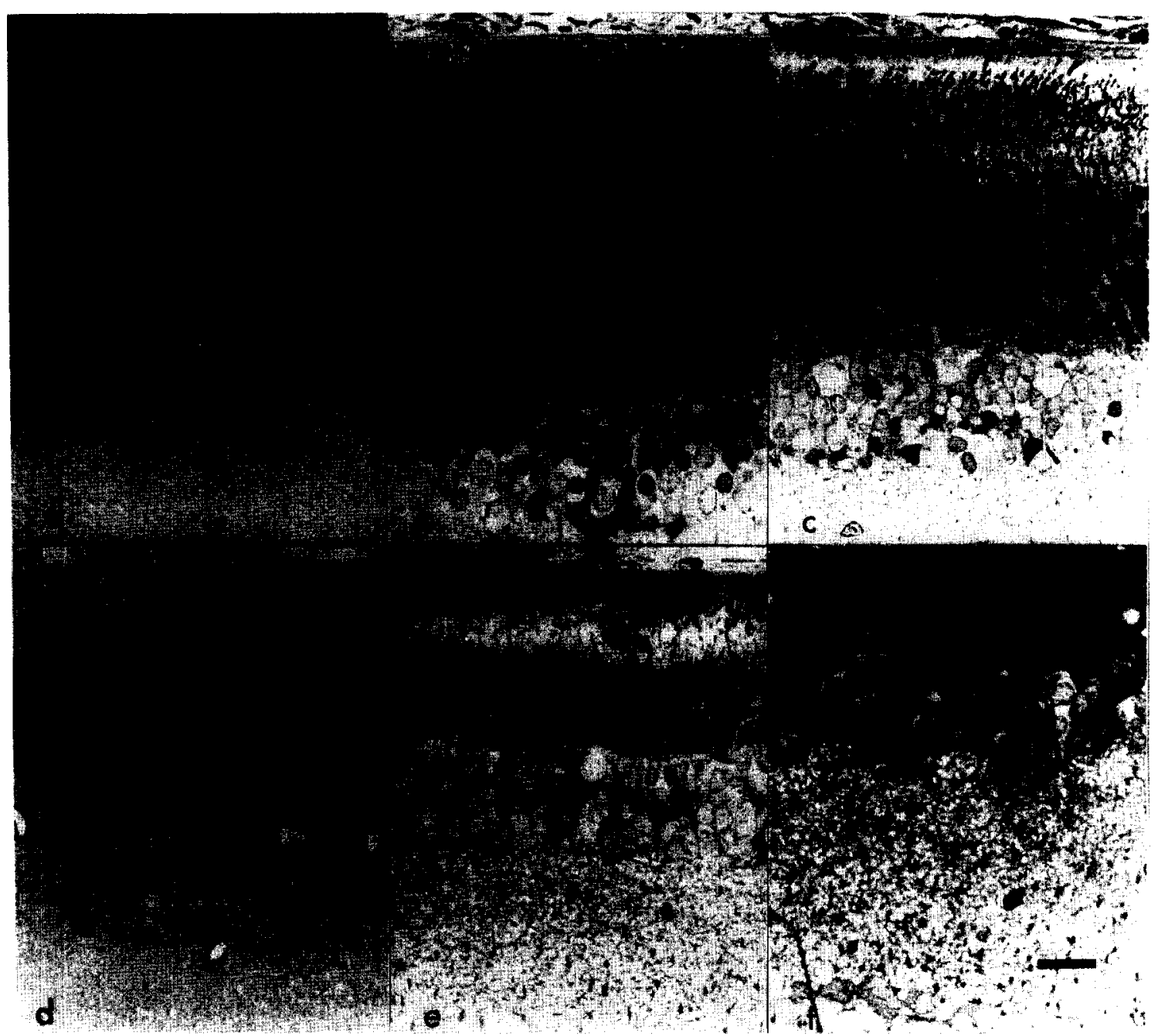

FIG. 1. Photomicrographs of the outer retina in horizontal section depict the criteria for the rating of outer segment damage, Outer Segment Loss, in light-exposed albino rat retinas. (a) A light micrograph of the healthy outer retina in horizontal section. Outer Segment Loss=0. $(\mathrm{RPE}=$ retinal pigmented epithelium; OS=outer segments; IS=inner segments; $\mathrm{ONL}=$ outer nuclear layer; $\mathrm{OPL}=$ outer plexiform layer; $\mathrm{INL}=$ inner nuclear layer; $\mathrm{IPL}=$ inner plexiform layer.) (b) Outer Segment Loss=1. The apical outer segments have a club- or whorl-like appearance and stain abnormally lightly. (c) Outer Segment Loss $=2$. The abnormal outer segments are not limited to the apical position but some order is still evident. (d) Outer Segment Loss $=3$. The outer segment layer is abnormal and highly disorganized with many palestaining whorls (arrows) which are damaged outer segments. (e) Outer Segment Loss $=4$. The outer segment layer is becoming thinner and is entirely composed of abnormal, damaged whorls (W). The inner segments are also highly disorganized. A nucleated cell body (M), probably a macrophage, appears among the damaged outer segments. (f) Outer Segment Loss $=5$, maximal loss. The entire outer segment layer, as well as most photoreceptor inner segments and nuclei, are irreversibly lost. In addition, in this case, the retinal pigmented epithelium is gone. A few surviving photoreceptor nuclei (arrows) can be seen. (Calibration bar $=20 \mu \mathrm{m}$ ).

analyzed statistically using the Student $t$-test for parametric data and the Mann-Whitney U-test for the nonparametric variable, Outer Segment Loss. Retinal ultrastructure after light exposure was also observed in electron micrographs, but was not quantitatively analyzed.

\section{RESULTS}

\section{Light Damage}

The bright light conditions clearly produced damage in the albino rat retina. Histological examination of the retinas revealed photoreceptor damage similar to that described in the literature. In many eyes the photoreceptor layers were highly abnormal and drastically depleted. In contrast, the inner retinal layers were essentially unaffected. Darkstaining nuclei which may have been chromatolytic were infrequently seen in the inner nuclear layer and ganglion cell layer.

The abnormal ultrastructure of damaged outer segments resembled sacs containing elongated vesicles, as described by Kuwabara and Gorn [9]. Light-damaged photoreceptor inner segments and nuclei were disrupted and vacuolated. In the most severe cases retinas were devoid of inner and outer segments, and occasionally the retinal pigment epithelium was also absent.

Frequently, nucleated cells were observed among the 


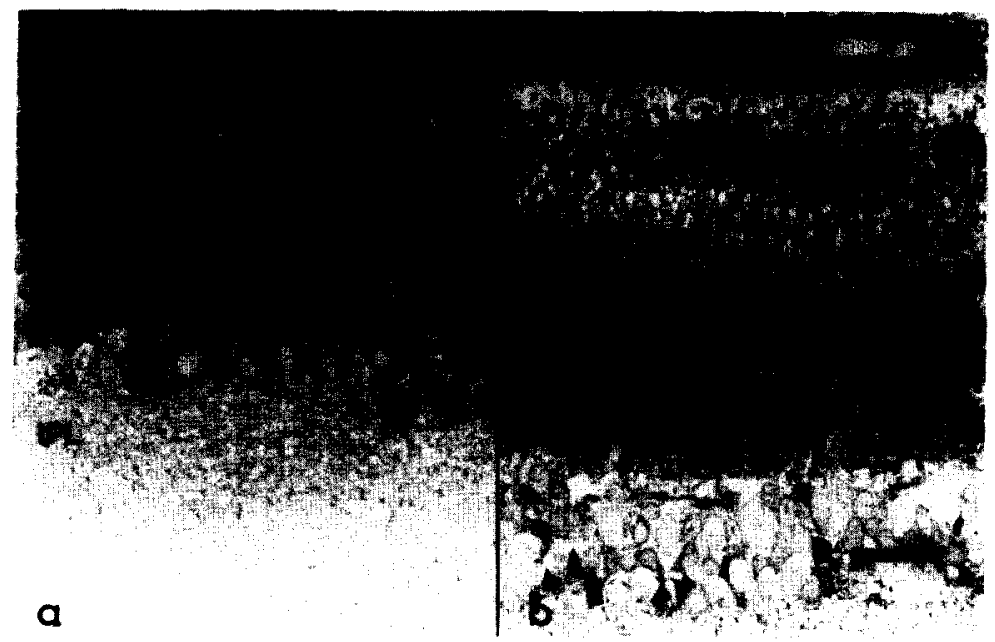

FIG. 2. (a) Photomicrographs of the outer retina in horizontal section from rats exposed to bright light in Experiment 1, after morning exposure. Severe photoreceptor loss is demonstrated by the thinning of the outer segment (OS), inner segment (IS), and outer nuclear layers. (RPE = retinal pigmented epithelium; $\mathrm{OPL}=$ outer plexiform layer; $\mathrm{INL}=$ inner nuclear layer; $\mathrm{IPL}=$ inner plexiform layer.) (b) Retinal damage is mild, after exposure in the afternoon, clearly less than that seen in morning-exposed retinas. Outer Segment Loss $=2$. $(O S=$ outer segments; IS=inner segments; Calibration bar $=20 \mu \mathrm{m}$.)

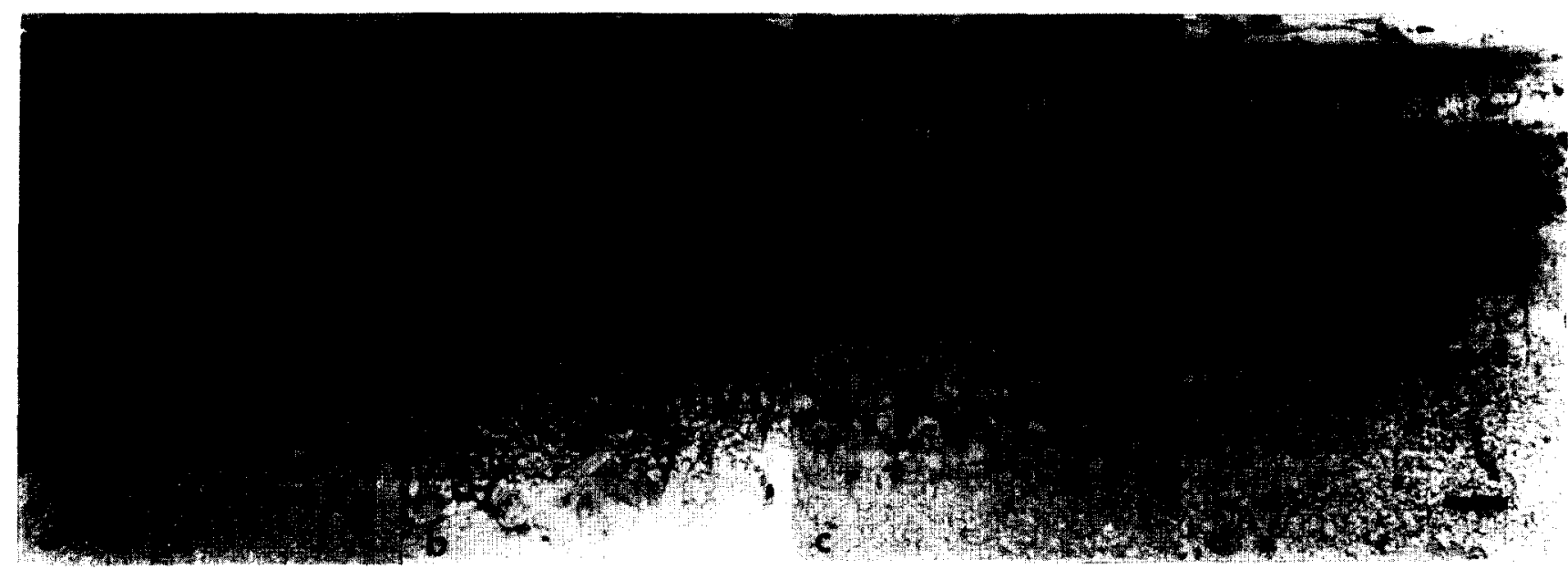

FIG. 3. (a) Photomicrographs of retinas from rats in Experiment 2. Control rats were sacriticed at the same tımes as light-exposed rats. Control retinas were not affected by time of sacrifice. (RPE=retinal pigmented epithelium; OS=outer segments; IS=inner segments; $\mathrm{ONL}=$ outer nuclear layer; $\mathrm{OPL}=$ outer plexiform layer; $\mathrm{INL}=$ inner nuclear layer; $\mathrm{IPL}=$ inner plexiform layer.) (b) The retina of a rat exposed to bright light in the morning shows severe loss of photoreceptors. Outer Segment Loss $=5$. The retinal pigmented epithelium is also gone. $(\mathrm{C}=$ choroid; $\mathrm{M}=$ macrophage-like cell; $\mathrm{ONL}=$ outer nuclear layer; $\mathrm{OPL}=$ outer plexiform layer; $\mathrm{INL}=$ inner nuclear layer.) (c) $\mathrm{The}$ retina of a rat exposed to bright light in the afternoon was less damaged than the morning exposure group. Outer Segment Loss=4. $(M=$ macrophage; $\mathrm{W}=$ abnormal, whorl-like outer segments.) (d) The retina of a rat exposed to bright light in the morning after 20 minutes light adaptation, shows intermediate photoreceptor loss between that seen in morning and afternoon exposure groups. Outer Segment Loss $=4$. (Calibration bar $=20$ $\mu \mathrm{m}$.

damaged outer segments (Fig. 1e). These cells were of an unknown origin and resembled macrophages. Their cytoplasm in electron micrographs sometimes contained phagosomal inclusion bodies. There was not a clear correlation of their presence with degree of damage. However, when a retina contained them, the animal's other retina always contained them also.

\section{Effect of Time of Day}

Retinas exposed early in the photoperiod sustained greater photoreceptor loss than retinas exposed in the afternoon. In Experiment 1, a clear-cut difference appeared between the morphological measurements of morning and afternoon-exposed retinas. As shown in Fig. 2, the photo- 
TABLE 2

MORPHOLOGICAL MEASUREMENTS AND OUTER SEGMENT LOSS IN THE RETINAS FROM EXPERIMENT 2

\begin{tabular}{|c|c|c|c|c|}
\hline \multirow[b]{3}{*}{$\begin{array}{l}\text { Retina } \\
\qquad \text { Mean }( \pm \text { S.E.M. })\end{array}$} & \multicolumn{4}{|c|}{ Treatment } \\
\hline & Morning & Light-Adapted & Afternoon & LD Only \\
\hline & $231.64 \quad( \pm 16.04)$ & $224.19 \quad( \pm 12.78)$ & $246.06 \quad( \pm 17.24)$ & $284.05^{*}+( \pm 10.75)$ \\
\hline Fiber Layer & $46.146( \pm 10.76)$ & $31.75( \pm 6.07)$ & $35.78 \quad( \pm 3.95)$ & $39.78 \quad( \pm 4.88)$ \\
\hline RPE & $5.60( \pm 2.11)$ & $9.95( \pm 1.62)$ & $10.24( \pm 0.66)$ & $9.31 \quad( \pm 0.58)$ \\
\hline $\begin{array}{r}\text { Apical RPE } \\
\text { Processes }\end{array}$ & $1.54( \pm 0.72)$ & $3.88( \pm 0.87)$ & $3.68^{*}( \pm 0.66)$ & $3.66( \pm 0.52)$ \\
\hline OS & $3.82( \pm 2.12)$ & $10.17^{*}( \pm 1.98)$ & $16.64^{*}( \pm 2.57)$ & $34.55 \quad( \pm 1.95)$ \\
\hline OS-IS & $7.07( \pm 3.07)$ & $17.44 *( \pm 3.16)$ & $27.62 *( \pm 3.87)$ & $51.28 \quad( \pm 2.34)$ \\
\hline ONL & $14.68( \pm 3.84)$ & $23.42( \pm 3.80)$ & $38.33 * \dagger( \pm 4.39)$ & $56.80 \quad( \pm 2.32)$ \\
\hline IPL & $58.80( \pm 3.41)$ & $66.92( \pm 3.32)$ & $56.86 \div( \pm 2.82)$ & $60.54 \quad( \pm 3.03)$ \\
\hline $\begin{array}{l}\text { Rows of ONL } \\
\text { Nuclei }\end{array}$ & $2.625( \pm 0.66)$ & $4.00( \pm 0.74)$ & $6.63^{* \dagger}( \pm 0.9)$ & $11.64( \pm 0.56)$ \\
\hline $\begin{array}{l}\text { OS Loss } \\
\text { Median (Range) }\end{array}$ & $5(4-5)$ & $4(4-5)$ & $4(4)$ & $1(0-2)$ \\
\hline
\end{tabular}

*Significantly different from Morning treatment.

tSignificantly different from Light-Adapted treatment.

Layer thickness in micrometers.

Light damage was determined by measuring the scleral-vitreal extent of photoreceptor layers in one-micron horizontal sections. Photoreceptor loss is indicated by thinning of outer retinal layers. The Morning group was exposed to light from 4:00-11:00 a.m. The Afternoon group was light-exposed from 11:00 a.m.-6:00 p.m. The greater loss of photoreceptors (OS, OS-IS, ONL) in the Morning group supports the prediction that light would be more damaging during rod dise shedding. The Light-Adapted group was in room illumination for 20 minutes before bright light exposure from 4:20 a.m.-11:20 a.m. Because their exposure also coincided with shedding, their greater damage relative to Afternoon-exposed retinas further supports the hypothesis. The "LD Only" group, unexposed controls, provide normal values for retinal layer thicknesses. S.E.M. =standard error of the mean; retina $=$ the entire retina, including the retinal pigmented epithelium; fiber layer $=$ optic fiber layer; RPE = retinal pigmented epithelium; apical RPE processes = processes of the RPE, measured between the RPE cytoplasm and the outer segment tips; $O S=$ outer segment layer; OS-IS=combined outer and inner segment layers; $\mathrm{ONL}=$ outer nuclear layer; $\mathrm{IPL}=$ inner plexiform layer; Rows of $\mathrm{ONL}$ nuclei=the number of photoreceptor cell bodies traversed by a single line; OS Loss = rating of outer segment damage, defined in the Method section.

receptor layers in the animals exposed in the morning were thinner, a direct result of loss of the photoreceptor outer segments, inner segments, and cell bodies. The combined thickness of outer and inner segments was $11.59( \pm 2.46) \mu \mathrm{m}$, the mean ( \pm the standard error of the mean), compared to $22.23( \pm 2.55) \mu \mathrm{m}$ for the retinas exposed in the afternoon. In the morning group, outer nuclear layer thickness averaged $28.36( \pm 4.75) \mu \mathrm{m}$. Afternoon-exposed retinas had an outer nuclear layer thickness of $43.6( \pm 4.09) \mu \mathrm{m}$. Measurements were significantly lower in the morning group than the afternoon group for the outer nuclear layer (Student $t$-test, $p=0.008$ ) and the combined outer and inner segment layer $(p=0.002)$.

Figure 2a shows an example of a retina light-damaged in the morning and can be compared to Fig. 2b, a light micrograph of an afternoon-exposed retina. The median value for Outer Segment Loss in the morning group was 4 and in the afternoon group was 3 , a significantly different score (Mann-Whitney $\mathrm{U}, p=0.001$ ).

The differential effect of the two times of exposure to bright light was replicated in Experiment 2. Again, morning exposure to bright light produced greater retinal damage than afternoon exposure. The outer segment layer in the morning retinas was $3.82( \pm 2.12) \mu \mathrm{m}$ compared to $16.64( \pm 2.57) \mu \mathrm{m}$ for afternoon retinas. Outer-plus-inner segments measured $7.07( \pm 3.07) \mu \mathrm{m}$ in morning retinas and $27.62( \pm 3.87) \mu \mathrm{m}$ in afternoon retinas. In the outer nuclear layer, rod cell body loss was also clearly worse after morning exposure. Outer nuclear layer thickness was $14.68( \pm 3.84) \mu \mathrm{m}$ in the morning group and $38.33( \pm 4.39) \mu \mathrm{m}$ in the afternoon retinas. In the morning group the outer nuclear layer was thinned to the point of extending through only $2.625( \pm 0.66)$ cell bodies in the basal-apical dimension. The afternoon group, with 6.63 $( \pm 0.9)$ nuclei through the outer nuclear layer thickness, had relatively less photoreceptor loss. All of these differences are statistically significant $(p<0.005)$. Experiments 1 and 2, therefore, confirmed that bright light is more effective in inducing light damage during the time of circadian disc shedding. Time of day of sacrifice had no effect on the six control rats, which had normal retinas (Fig. 3a). Morphological measurements on these retinas provide a baseline (" $\mathrm{LD}$ Only" in Table 2) to evaluate the amount of damage in the eyes exposed to bright light. From these data, it appears that normally the outer nuclear layer is $56.8( \pm 2.32) \mu \mathrm{m}$ thick with a vertical complement of $11.64( \pm 0.56)$ nuclei in this thickness. The morning group in Experiment 2 had a 75-80\% loss in outer nuclear layer thickness, while afternoon retinas only sustained an outer nuclear layer loss of $30 \%$. In the 
morning group, but not the afternoon group, the thickness of the retina was significantly less, $231.64( \pm 16.04) \mu \mathrm{m}$, than in controls $284.05( \pm 10.75) \mu \mathrm{m}(p<0.02)$, due to photoreceptor loss.

The overall effect of bright light in Experiment 2 was stronger than in Experiment 1. That is, animals exposed in the morning in Experiment 2 had greater photoreceptor damage than rats exposed in the morning in Experiment 1 , and the same relationship held for retinas light-damaged in the afternoon. For example, median Outer Segment Loss in Experiment 1 was 4 (Fig. 2a) and in Experiment 2 was 5 (Fig. $3 b)$ for retinas of the morning groups. A median score of 3 was recorded for Outer Segment Loss in Experiment 1 afternoon-exposed retinas (Fig. 2b), and a score of 4 in the Experiment 2 afternoon exposure group (Fig. 3c).

\section{Effect of Light Adaptation}

The group of rats which were light-adapted for 20 minutes before bright light exposure showed an intermediate degree of retinal damage (Fig. 3d). The light-adapted retinas were significantly different from the morning exposure group on measures of outer and inner segment layers, the combined thickness of which was $7.07( \pm 3.07) \mu \mathrm{m}$ in the morning retinas compared to $17.44( \pm 3.16) \mu \mathrm{m}$ in the light-adapted retinas $(p=0.04)$. On these measures, the afternoon group was not significantly different from the light-adapted group.

Conversely, the light-adapted retinas showed significantly worse rod cell body depletion than the afternoon group. Light-adapted retinas had only $4.00( \pm 0.74)$ rows of nuclei through the thickness of the outer nuclear layer, which measured $23.42( \pm 3.80) \mu \mathrm{m}$. The afternoon group averaged $38.33( \pm 4.39) \mu \mathrm{m}$ in outer nuclear layer thickness $(p=0.02)$, and $6.63( \pm 0.9)$ rows of nuclei $(p=0.04)$. The morning eyes had worse outer nuclear layer loss than lightadapted retinas but to a degree which was nonsignificant. Like the morning group, light-adapted retinas were significantly thinner, $224.19( \pm 12.78) \mu \mathrm{m}$, than controls $(p=0.01)$, due to severe loss of photoreceptors.

Therefore, it appears that adaptation to the room lighting by the afternoon rats was a factor contributing to their reduced vulnerability to damaging light. However, the protection afforded by light adaptation was not sufficient to account for the morning-afternoon difference.

The inner plexiform layer thicknesses were measured in the retinas as a check on orientation of the vertical sections, since there is no evidence that this layer is affected by light damage. Some sort of check becomes necessary, because outer segments are abnormal or lost in damaged eyes, and no other visual guide to orientation is available. In Experiment 2 , the inner plexiform layer of the light-adapted group was thicker than that of afternoon retinas $(p<0.05)$. Therefore, it is possible that improper orientation, a deviation from the intended true horizontal plane, inflated some of the measurements of retinal layers in the light-adapted group. Alternatively, light damage produced this change, in spite of the otherwise normal histological appearance of the inner plexiform layer in all eyes.

\section{Temperature}

Temperature was recorded several times a day and was $27^{\circ} \mathrm{C}( \pm 0.07)$. In addition, for 21 days during Experiment 2 a continuous record was made with a thermistor-driven pen. The results showed that the muffin fans gave adequate venti- lation. When lights were off in the wooden light box, the temperature inside was equal to the room temperature. When lights were on, the temperature was raised within one min to $0.44^{\circ} \mathrm{C}$ higher than the room temperature where it remained as long as the lights were on. Therefore, temperature was not different during the morning and afternoon exposures, and cannot explain the findings of greater light damage in the morning exposure group. Nor is the greater degree of damage in Experiment 2 relative to Experiment 1 explained by a temperature change.

\section{DISCUSSION}

Based on the hypothesis that bright light damages rods by disturbing outer segment turnover, it was predicted that photic damage would be more severe during outer segment disc shedding. This prediction was tested experimentally by comparing the retinal damage produced by light exposure in the morning (4:00 a.m.-11:00 a.m.) to the damage produced by exposure to the same conditions in the afternoon (11:00 a.m. $-6: 00$ p.m.). The morning exposure completely included the times of circadian outer segment disc shedding, i.e., one-to-three hours after light onset [15]. The afternoon exposure took place after the daily peak in shedding had returned to baseline [11]. Had no differences been seen in the degree of damage produced by the two treatments, the prediction would be disproved and the hypothesis discredited. However, in the event, morning exposure to bright light destroyed twice as many photoreceptors as afternoon exposure supporting the hypothesis.

The theory that abnormal outer segment turnover is the mechanism of photic damage is consistent with the demonstration of an early effect on retinal pigment epithelium function during bright light exposure $[6,7]$, and with the blocking of circadian shedding by bright light [26]. However, it is equally plausible that diurnal changes in pigment epithelium function which are unrelated to outer segment phagocytosis and renewal underlie the effect of time of day. Since O'Steen has clearly shown hormonal modulation of light damage $[16-18,20]$ it is also possible that a diurnal change in endogenous hormones mediates retinal sensitivity to light. Our use of a 14:10 light-dark cycle differs from the commonly reported 12:12 light-dark cycle [12]. However, we have observed the circadian increase in large phagosomes in retinas of both mice and rats from our animal rooms (unpublished data).

Because the morning exposure began at light onset, the two groups differed in an additional factor. The morning group was dark-adapted at the onset of bright light, while the afternoon group was light-adapted to room lighting at bright light onset. To exclude the possibility that light adaptation was the factor responsible for the differential effect of time of exposure, a group of animals in Experiment 2 were in the room illumination for 20 minutes before their daily bright light exposure began. Twenty minutes is well above the period necessary to achieve steady-state light adaptation (bleaching) to the room lighting [2]. As reported above (in the Results section), these animals sustained an intermediate degree of photoreceptor loss. The light adaptation was effective in reducing damage, but light-adapted retinas were not equivalent to the afternoon retinas. The results demonstrate that morning exposure enhances light damage regardless of photoreceptor adaptation state.

The protection by light adaptation recalls the "state of cell adaptation to light' that Noell and Albrecht [15] found to 
be critical in determining sensitivity to light damage. They showed that rats raised in cyclic light conditions were less susceptible to photic damage than dark-reared rats [15]. All the subjects in the present experiments were reared in cyclic lighting, but differed in short-term photoreceptor light adaptation. The present data thus extend Noell's demonstration that retinal sensitivity to damaging light depends on prior lighting conditions.

While the differential effect of morning exposure was replicated, the same light conditions were less damaging in Experiment 1 than in Experiment 2. Measured light intensity was $2.16 \mathrm{log} \mathrm{ftc}$ in Experiment 1 and $2.14 \mathrm{log} \mathrm{ftc}$ in Experiment 2, a slight decrement at the limits of the photometer's accuracy. Temperature was not changed, and therefore cannot account for the disparity. The age of subjects at the beginning of bright light exposure in Experiment 1 was 105 days compared to 74 days in Experiment 2 . Since only adult animals were used, no age effect was anticipated [19]. Longterm light exposure effects have been shown in two- and three-year-old albino rats [27]. More experimentation is merited to determine whether the present result is due to aging. Seasonal differences in susceptibility to light damage might explain the observation since Experiment 1 was conducted in February, Experiment 2 in June. Note that in each experiment, animals were compared only at the same age and at the same time of year, and that the primary effect of time of day on severity of damage was demonstrated in each experiment.

These experiments studied retinal sensitivity to light damage with fairly subtle environmental changes. Fifkova [3] likewise found photoreceptor loss with minor alterations of the photoperiod. Histological measurements after two months in 16:8 and 14:10 light-dark cycles demonstrated thinning of the outer retina relative to animals kept in a 12:12 light-dark cycle [3]. Similar methods should have future applications to problems of photic damage. The present demonstration that bright light exposure at the time of shedding and phagocytosis is more effective in producing retinal damage than exposure at a time of phagosomal quiescence raises an important question. Is this effect due to the action of light at the pigment epithelium, at the photoreceptors, or at a central site which hormonally controls shedding and phagocytosis?

\section{REFERENCES}

1. Anderson, R. E., L. M. Rapp and R. D. Weigart. Lipid peroxidation and retinal degeneration. Curr Eye Res 3: 223, 1984.

2. Dowling, J. E., Neural and photochemical mechanism of rod adaptation in the rat. $J$ Gen Physiol 46: 1287, 1963.

3. Fifkova, E. Plastic and degenerative changes in visual centers. Adv Psychobiol 2: 59, 1974.

4. Fisher, L. J. Development of synaptic arrays in the inner plexiform layer of the neonatal mouse retina. I Comp Neurol 187: 359, 1979.

5. Grignolo, A., N. Orzalesi, R. Castellazzo and P. Vittone. Retinal damage by visible light in albino rats, an electron microscope study. Ophthalmologica 157: 43, 1969.

6. Hansson, H. A. A histochemical study of cellular reactions in rat retina transiently damaged by visible light. Exp Eye Res 12: 270,1971 .

7. Hansson, H. A. A histochemical study of oxidative enzymes in rat retina damaged by visible light. Exp Eye Res 9: 285, 1968.

8. Hoffmann, J. C. The influence of photoperiods on reproductive functions in female mammals. Handbk Physiol (Section 7, Endocrinology) 2 (Part 1): 57, 1973.

9. Kuwabara, T. and R. Gorn. Retinal damage by visible light-an electron microscopic study. Ophthalmologica 157: 43, 1969.

10. LaVail, M. M. Eye pigmentation and constant light damage in the rat. In: The Effects of Constant Light on Visual Processes, edited by T. P. Williams and B. N. Baker. New York: Plenum, 1980 , p. 357.

11. LaVail, M. M. Rod outer segment disc shedding in rat retina: relationship to cyclic lighting. Science 194: 1071, 1976.

12. LaVail, M. M. Circadian nature of rod outer segment disc shedding in the rat. Invest Ophthalmol Vis Sci 19: 1268, 1980.

13. Noell, W. K. Aspects of experimental and hereditary retinal degeneration. In: Biochemistry of the Retina, edited by C. N. Graymore. New York: Academic Press, 1965, p. 51.

14. Noell, W. K., V. S. Walker, B. S. Kang and S. Berman. Retinal damage by light in rats. Invest Ophthalmol 5: 450, 1966.

15. Noell, W. K. and R. Albrecht. Irreversible effects of visible light on the retina: role of vitamin A. Science 172: 76, 1971.
16. Olafson, R. P. and W. K. O'Steen. Hormonal influences on photoreceptor damage: The pituitary and ovaries. Invest Ophthalmol Vis Sci 15: 869, 1976.

17. O'Steen, W. K. Ovarian steroid effects on light-induced retinal photoreceptor damage. Exp Eye Res 25: 361, 1977.

18. O'Steen, W. K. and S. L. Kraeer. Effects of hypophysectomy, pituitary gland homogenates and transplants, and prolactin on photoreceptor destruction. Invest Ophthalmol 16: 940, 1977.

19. O'Steen, W. K., K. V. Anderson and C. R. Shear. Photoreceptor degeneration in albino rats: Dependency on age. Invest Ophthalmol Vis Sci 13: 334, 1974.

20. O'Steen, W. K. Hormonal influences on retinal photodamage. In: The Effects of Constant Light on Visual Processes, edited by T. P. Williams and B. N. Baker. New York: Plenum, 1980, p. 29.

21. O'Steen, W. K. and R. B. Lytle. Cellular disruption and phagocytosis in photically induced retinal degeneration. $A m J$ Anat 130: 227, 1971.

22. O'Steen, W. K., C. R. Shear and K. V. Anderson. Retinal damage after prolonged exposure to visible light. A light and electron microscopy study. Am J Anat 134: 5, 1972.

23. Rapp, L. M. and T. P. Williams. A parametric study of retinal light damage in albino and pigmented rats. In: The Effects of Constant Light on Visual Processes, edited by T. P. Williams and B. N. Baker. New York: Plenum, 1980, p. 135.

24. Rusak, B. and I. Zucker. Biological rhythms and animal behavior. Annu Rev Psychol 26: 137, 1975.

25. Shear, C. R., W. K. O'Steen and K. V. Anderson. Effects of short-term low intensity light on albino rat retina. An electron microscopic study. Am J Anat 138: 127, 1973.

26. Teirstein, P. S., A. I. Goldman and P. J. O'Brien. Evidence for both local and central regulation of rat rod outer segment disc shedding. Invest Ophthalmol Vis Sci 19: 1268, 1980.

27. Weisse, I. and H. Stotzer. Age- and light-dependent changes in the rat eye. Virchows Arch [A] 362: 145, 1974. 